\title{
El concepto de "partido político" y los mandarines chinos en el exilio: concepto y experiencia entre China y las Américas (fines del siglo XIX-principios del XX)
}

\section{The Concept of "Political party" and Chinese Mandarins in Exile: Concept and Experience between China and the Americas}

\section{Pablo Ariel Blitstein ${ }^{1}$ \\ CRH-EHESS (Francia)}

Recibido: 27-06-18

Aprobado: 26-07-18

\section{Resumen}

La vasta bibliografía que ha sido dedicada al concepto de "partido político" suele dejar de lado la historia no-occidental de este concepto (con excepción de Rusia), y los trabajos que la abordan suelen limitarla a un marco nacional. Las páginas que siguen proponen remediar en parte esta carencia. Con el objeto de mostrar la complejidad de los procesos de transmisión y producción de conceptos políticos en los albores del siglo XX, este artículo aborda un breve episodio en la historia global del partido: es la historia del concepto de $d a n g$ - un término de época imperial que a partir de fines del siglo XIX fue asociado a la noción de "partido" del discurso político euro-americano - y de las peripecias transcontinentales de este concepto entre el Este asiático y las Américas. La historia en cuestión tiene por objeto un dang reformador de fines del siglo XIX. Este dang nació en el imperio Qing en 1895 como reacción a la Guerra Sino-Japonesa y en 1898 logró consolidarse como una facción de la corte del emperador Guangxu. Expulsado

\footnotetext{
${ }^{1}$ (pabloarielblitstein@gmail.com). Doctor en Historia y profesor del Institut Nationale de Langues et Civilisations (INALCO) de Paris donde también obtuvo su Maestría en estudios chinos. Actualmente se desempeña también como profesor e investigador del CEMECH (Escuela de Humanidades/ UNSAM). Ha publicado artículos y traducciones en el campo de la literatura romana antigua y la historia china.
} 
por un golpe de palacio, el dang reformador fue reorganizado en 1899 bajo la forma de una asociación en el exilio, y mientras sus dirigentes lo extendían a diferentes partes del mundo - de Asia a las Américas, de Japón a Malasia, de Australia al Perú-, el dang comenzó paulatinamente a asociarse al concepto de "partido político" en el mundo occidental. La reconstrucción de esta historia nos permitirá elucidar no sólo el modo en que el concepto de partido político en China estuvo asociado a las discusiones y decisiones que el establecimiento de esta organización transcontinental requirió de sus dirigentes y miembros, sino, más en general, el modo en que se articulan producción de conceptos y experiencia social.

Palabras-clave: reformadores chinos; partido político; conceptos políticos; dinastía Qing; Américas

\begin{abstract}
Most of the research on the concept of "political party" often neglects the non-Western history of this concept (with the exception of Russia), and the scholars who deal with it usually remain within the limits of a national framework. We intend to compensate at least in part for this lack. With the aim of disclosing the complexity of the production and transmission of concepts at the beginning of the 20th century, we will deal with a brief episode in the global history of the political party. We will study the history of dang - an imperial term increasingly associated with the Euro-American concept of "party" in the late 19 th and early 20th centuries - and the transcontinental hazards of this concept between East Asia and the Americas. We will more particularly focus on the dang of late 19th century Chinese reformers. This dang emerged in in the Qing empire in 1895 after the Sino-Japanese war and in 1898 it managed to become a faction at the court of the emperor Guangxu. After a palace coup expelled it from China, the dang of the reformers was reorganized as an association in exile, and while its leaders extended it to different parts of the world - from Asian to the Americas, from Japan to Malasia, from Australia to Peru- , the dang was increasingly associated with the concept of "political party" in the Western world. This history will help us elucidate the way in which the concept of political party in China was related not only to the discussions and decisions of the leaders of this transcontinental association, but also, and more generally, to the way in which the production of concepts is related to social experience.
\end{abstract}

Key-words: Chinese reformers; political party; political concepts; China; Qing dynasty; Americas. 
La noción de "partido político" nada tiene en sí misma de evidente, ni en la historia europea, ni en la historia de Asia o de las Américas. Del partido"facción" al "partido único", del "partido de masas" al "partido de opinión”, la institución política que llamamos "partido" suele contrastar con lo que a menudo se considera el "tipo ideal" de partido político: su manifestación en el marco de instituciones representativas. Este tipo ideal tiene una historia, una historia mundial. Tiene una historia mundial no sólo en el sentido de que cada historia individual es también, forzosamente, un breve episodio en la historia general del globo terráqueo; lo es también en el sentido de que las redes de conexiones que han hecho posible el concepto moderno de "partido" no siguen -nunca lo han hecho- la geografía jurídica de los estados-nación. Los partidos políticos de distintas partes del mundo, ya sea en sus ideologías, ya en sus estructuras, nunca dejaron de observarse mutuamente, de imitarse, de diferenciarse o de intercambiarse prácticas y discursos; muchos incluso coordinan sus acciones y sostienen mutuamente sus militantes respectivos más allá de las fronteras. Todos estos fenómenos han sido y siguen siendo constitutivos de la historia de los partidos políticos en el mundo.

Y sin embargo, a pesar de la dimensión mundial que posee la geografía social y intelectual del partido político contemporáneo, la vasta bibliografía que le ha sido dedicada - tanto a su concepto como a los grupos que lo constituyen - se concentra ante todo en la historia europea (Rusia incluida). Salvo en las historias que tienen por objeto el Este asiático, apenas se menciona que una buena parte de la historia de los partidos políticos ha transcurrido fuera de Europa y fuera de América del Norte. Las páginas que siguen proponen remediar al menos en parte esta carencia. Proponen, a modo de ilustración, abordar una breve peripecia de esa historia no europea del partido: es la historia del concepto de dang y de los grupos que en China llevaron este nombre. La historia de este término chino es vasta y compleja. A partir de fines del siglo XIX, en un proceso de interconexión semántica con la palabra "partido" de las lenguas europeas contemporáneas, dang quedó definitivamente asociado al discurso político europeo, y las evoluciones semánticas de ambos términos son desde ese entonces inseparables; pero dado que estaba marcado por su antiguo protagonismo en el lenguaje jurídico, político e historiográfico del imperio, esta palabra - cuyo significado era similar al de nuestra palabra "facción"adoptó una serie de connotaciones que son indisociables de las experiencias políticas del mundo sino-parlante ${ }^{2}$. De este modo, las experiencias que el mundo

2 Esta dimensión espacial de la producción de ideas e instituciones políticas no ha sido suficientemente tenida en cuenta por la historia de las ideas y los conceptos políticos. Quentin Skinner fue en esta dirección cuando recurrió a la lingüística pragmática para reconstruir el significado de los discursos políticos. A pesar de las diferencias metodológicas, los investigadores se asociaron con el proyecto Geschichtliche Grundbegriffe de Reinhart Koselleck, Otto Brunner y Werner Conze también se han ocupado de restaurar el contexto social en el que los conceptos políticos adquieren significado. Ver Melvin Richter, The History of Political and Social Concepts: A Critical Introduction, New York- 
occidental ponía bajo el nombre de partido o de sus variantes entraron, de modo fragmentario y refractario, en el campo semántico de la palabra dang; y viceversa, las nuevas experiencias asociadas a la palabra dang contribuyeron a su vez - entre otros por medio del discurso historiográfico- a la extensión del campo semántico del "partido" en una y otra parte del globo.

Una buena parte de los trabajos sobre la historia de dang se ha concentrado en las evoluciones políticas en China misma o en el Este asiático. De los salones de estudio a fines del imperio al régimen partido único bajo el Guomin dang, la evolución del dang aparece asociada ya sea a instituciones -en especial la institución del estado-nación-, ya sea a grupos instalados en las grandes ciudades del territorio chino o japonés -sobre todo mandarines, comerciantes, hombres de letras y estudiantes. Aquí querría en cambio recuperar un itinerario menos esperado, pero igualmente significativo para reconstruir los procesos complejos de transmisión y producción de conceptos políticos en los albores del siglo XX: se trata del dang "reformador", que se proponía transformar la antigua monarquía imperial de Qing en una monarquía nacional, constitucional y parlamentaria. Este dang, impulsado por Kang Youwei y otros mandarines de renombre (mejor o peor posicionados en las instituciones imperiales), cobró forma forma en 1895 como reacción a la Guerra Sino-Japonesa, y en 1898 logró consolidarse como una facción de la corte del emperador Guangxu. A partir de ese momento, el dang reformador ya no tuvo como marco un "estado-nación”. Reorganizado en 1899 bajo la forma de una asociación en el exilio ${ }^{3}$, Kang Youwei y otros dirigentes reformadores extendieron su organización a diferentes partes del mundo, de Asia a las Américas, de Japón a Malasia, de Australia al Perú. Los partidos políticos en China deben una parte de su historia a las discusiones y decisiones que el establecimiento de esta organización transcontinental requirió de sus dirigentes y miembros. Como suele ser el caso, ni su historia política se reduce a su presencia en un Estado, ni su historia social se reduce a las fronteras jurídicas de un territorio. Ambas historias, a veces imposibles de distinguir, remontan a complejos de relaciones con frecuencia invisibles, y sólo algunos indicios nos permiten ver cómo las ideas y las prácticas reemergen transfiguradas en geografías físicas y sociales a veces inesperadas para el historiador.

Oxford, Oxford University Press, 1995, p. 9-25. A partir de un análisis de estas tendencias, Jacques Guilhaumou llamó la atención sobre la importancia de restaurar el contexto lingüístico y práctico de los "usos conceptuales"; más recientemente, Frédérique Matonti ha propuesto una "historia social de ideas políticas" que estudia la convergencia de diferentes mundos sociales en la producción de ideas políticas. Ver, por ejemplo, J. Guilhaumou, "De l'histoire des concepts à l'histoire linguistique des usages conceptuels", Genèses, n 38, 2000, p. 105-11; F. Matonti, "Plaidoyer pour une histoire sociale des idées politiques", Révue d'histoire moderne et contemporaine, vol. V, n 59, 2012, p. 85-104. En este artículo, nos inscribimos en la continuidad de estos intentos y nos centramos en la localización de las experiencias sociales que están en el origen de nuevos conceptos políticos, en este caso, el de "partido político".

${ }^{3}$ Wu Tianren, Kang Youwei xiansheng nianpu, Taipei, Yiwen yinshu guan, 1994, p. 273 (desde aquí, «xiansheng nianpu»).

Araucaria. Revista Iberoamericana de Filosofía, Política, Humanidades y Relaciones Internacionales, año 20, ${ }^{\circ} 40$. Segundo semestre de 2018. Pp. 159-184. ISSN 1575-6823 e-ISSN 2340-2199 doi: 10.12795/araucaria.2018.i40.07 


\section{EI dang y la corte}

El mundo de los dang es el mundo del imperio. Al menos en su forma tardía, es el mundo de la monarquía imperial, de su corte, de una "nobleza" de dimensiones y privilegios variables, de un estrato de ministros y funcionarios regulares (con su personal de apoyo) y, en las localidades y las ciudades, de una clase de mandarines que proveía los candidatos a formar parte de la administración. En este mundo, no había ni parlamento ni elección "de abajo hacia arriba", sino administración y selección de mandarines "de arriba hacia abajo". Los dang imperiales se desarrollaron en este contexto institucional.

La palabra dang, noción clave en este mundo monárquico, tenía ante todo connotaciones negativas. Como las palabras derivadas del latín factio en varios idiomas europeos ${ }^{4}$, dang nombraba desde principios del imperio cualquier grupo de funcionarios y ministros formado alrededor de intereses comunes y de dependencias mutuas. Durante la dinastía Qing, sobre la base de una percepción negativa del final de la dinastía Ming, los grupos acusados de "constituir un dang y buscar el beneficio personal" (jiedang yingsi) eran perseguidos sistemáticamente. Esta frase aparece una y otra vez en la historiografía imperial y en la literatura jurídica. En los Estatutos de la dinastía Qing con sus precedentes (Da Qing huidian shili) -compendio de normas y precedentes administrativos y jurídicos de la dinastía imperial- la expresión "constituir un dang" se aplica a bandidos, rebeldes, mandarines agitadores, funcionarios sediciosos o todo tipo de grupo que pusiera en peligro la estabilidad de la sociedad imperial ${ }^{5}$. Si existía la sospecha de que la acción individual de un ministro respondía a una acción colectiva organizada, las personas implicadas corrían el riesgo de ser acusadas de constituir un dang y de sufrir diferentes tipos de penas según la gravedad del delito. Cierto es que algunos dang podían de tanto en tanto tener connotaciones positivas. Cuando el emperador estaba en peligro, cuando el Cielo parecía haberle retirado su favor o cuando un dang "sedicioso" controlaba la corte, un grupo mandarines con o sin puesto podían constituirse en facción y luchar contra los elementos "corruptos" en la corte y la administración; en ese caso, las lealtades mutuas entre mandarines, cristalizadas en un dang, eran un requisito para la regeneración del imperio. La historiografía de época imperial está llena de ejemplos de este tipo. Los

\footnotetext{
${ }^{4}$ Klaus von Beyme, "Partei, Faktion", in Brunner, Otto, Werner Conze, y Reinhart Koselleck (ed.) Geschichtliche Grundbegriffe. Historisches Lexikon zur politisch-sozialen Sprache in Deutschland, Stuttgart: Klett-Cotta, 1972-1997, p. 677.

${ }^{5}$ Es lo que puede apreciarse con una mirada rápida a la ed. de 1899 de los estatutos. Por ejemplo, en el juicio de 1726 de Nian Gengyao, estudiado por Otani Toshio, la acusación contiene esta misma frase. Ver Otani Toshio, "Nen Kôgyo danzai jiken no seijiteki haikei”, dans Shindai seiji shisô shi no kenkyû, Tôkyo : Kyûko Shoin, 189, citado en Murata Yujiro, "Dynasty, State, and Society: The Case of Modern China," in J. Fogel, J. y Zarrow, P., Imagining the People: Chinese Intellectuals and the Concept of Citizenship, 1890-1911, Armonk: M. E. Sharpe, 1997, 127.
} 
relatos de fines de reino suelen celebrar dang de funcionarios y ministros que se oponen al rumbo desviado de la dinastía reinante, y estos dang, según la mirada del historiador, anuncian en sus acciones la futura llegada de un monarca más justo. Pero incluso los dang "justicieros" se presentaban como el síntoma de un mal profundo: "los dang son una enfermedad peligrosa para el reino", decía Tang Zhen en el siglo XVII. "Si no se la cura, está condenado a perecer"6.

Esta connotación negativa, que explica las sucesivas prohibiciones de los dang a lo largo de la historia imperial, estaba ligada a un concepto político clave: la idea de que la cohesión política del imperio estaba fundada en la "lealtad" al emperador. Los ministros, en principio "servidores" del emperador, tenían que mostrar una dedicación leal a su "señor" más allá del beneficio que podían obtener. Cualquier lealtad intermedia, ya fuera hacia un superior o hacia un par, no debía en ningún modo ser más fuerte que la lealtad que le debían al emperador. Las razones prácticas de esta exigencia son evidentes. Si los funcionarios creaban facciones, existía el riesgo de que los dang se bloquearan entre sí y paralizaran la comunicación administrativa, que el ingreso fiscal quedara en manos de un grupo provincial, que las milicias locales quedaran a la merced de magistrados sediciosos o que los reclutamientos y promociones quedaran en manos de un pequeño grupo. Cualquier ruptura en la unidad fundamental de la monarquía representaba, según los criterios de la corte, un peligro para el imperio. Y si el imperio se encontraba en peligro, era también el orden cósmico que podía desbandarse: porque según la cosmología imperial, el emperador -el "hijo del Cielo"- tenía por misión mantener en harmonía el cosmos, y la administración era su instrumento principal para conseguir este objetivo.

Durante la primera década del siglo XX, los mandarines reformadores, y en particular los reformadores nacionalistas, crearon sobre esta base tradicional una nueva forma de dang. Este grupo de mandarines reformadores, algunos de ellos bien posicionados en la administración imperial, otros, como Kang Youwei, asentados en un puesto subalterno, y otros finalmente sin puesto alguno, lograron una cierta visibilidad después de la Guerra Sino-japonesa de 1895. La guerra provocó en efecto un nuevo impulso reformador: la derrota frente al Japón de Meiji parecía haberle demostrado a la dinastía que sus medidas de "auto-fortalecimiento", inspiradas por la generación precedente de mandarines reformadores, habían fallado, y que sólo una serie de reformas más radicales podría permitirle alcanzar el nivel de los logros de Meiji. En ese contexto, un grupo de mandarines, en especial Kang Youwei y sus discípulos, propusieron una reforma integral de la monarquía y de la sociedad imperial. Parte de las propuestas, como el fortalecimiento económico y militar, se inspiraban en las ideas de la generación precedente de reformadores; otras,

\footnotetext{
${ }^{6}$ Tang Zhen, Qianshu jiaozhu, Changsha: Yuelu shushe, p. 214.
} 
más o menos cercanas al modelo de Meiji, iban más allá: la apertura de una asamblea nacional, una reforma de la administración y de las instituciones escolares y la codificación de un nuevo tipo de legislación ${ }^{7}$. Durante la llamada "reforma de los cien días," en 1898, el emperador Guangxu escuchó con atención a este grupo de reformadores; incluso implementó algunas de sus propuestas, sobre todo la creación de una universidad imperial y la reforma de los concursos de reclutamiento de mandarines. El emperador tenía sólo 26 años de edad en ese entonces. Pero en septiembre de ese mismo año, un grupo de ministros "conservadores" (en general reformadores de la generación precedente), apoyados por la emperatriz viuda Cixi, se apoderaron del palacio por la fuerza, encerraron al emperador Guangxu en el palacio y ejecutaron a una buena parte de los reformadores. Kang Youwei y su discípulo Liang Qichao, dos de las principales figuras del grupo, lograron escapar a Japón y Canadá. Esta coyuntura representó un momento propicio para cargar el dang de connotaciones hasta entonces desconocidas en el mundo imperial.

Es verdad que el concepto de dang preservó muchas de sus connotaciones tradicionales en el mundo mandarinal, tanto del lado reformador como del lado conservador. Cuando sus adversarios acusaban a los reformadores de haber creado un dang, el uso de esta palabra no se alejaba demasiado del que le daba el discurso tradicional de la dinastía: era un término negativo que sugería la idea de facción ilegítima. Así, durante la represión de 1898, Kang Youwei fue acusado de "constituir un dang y perseguir intereses personales" (jiedang yingsi) ${ }^{8}$. Los reformadores, por su parte, retomaron desde el principio la tradición del dang "justiciero". Por ejemplo, en una ocasión en que caminaba con sus discípulos por la gruta de Longyin en Guilin, Kang Youwei se detuvo a leer la llamada "Estela de los dang" que Cai Jing (1047-1126), bajo los Song (960-1279), había hecho escribir en contra del dang de Sima Guang (1019-1086). Al leerla, Kang decidió agregarle un colofón": "los miembros del dang de los Han posterior, la 'proscripción de los dang' en la era Yuanqing de los Song del sur y el dang de Donglin bajo los Ming: eso da en total cuatro [dang] si contamos éste, [el dang de Sima Guang]. Cao Jie (? -181), Cai Jing, Han Tuozhou (1152-1207), Wei Zhongxian (1568-1627) atacaron los dang; Li Ying (? -169), Sima Guang, el Maestro Zhu [Xi] (1130-1200), Gao [Panlong] (1562-1626) y Gu [Xiancheng] (1550-1612) fueron parte de un dang. [Pero] la posteridad no ha forzosamente hecho de estos hombres de dang el objeto de un tabú. ¿Acaso no les gustaría convertirse en Li Ying, Sima Guang, Maestro Zhu [Xi], Gu Xiancheng? [¿A quién le gustaría] seguir los pasos de [los eunucos] Hou Lan (? -172) y Wei

${ }^{7}$ Xiansheng nianpu,187. En agosto de 1898, Kang solicitó una constitución y un parlamento.

${ }^{8}$ Qingshi gao, 24.926.

9 Sobre Cai Jing y esta estela, ver Ari D. Levine, Divided by a Common Language: Factional Conflict in Late Northern Song China, Honolulu : University of Hawaii Press, 2008, p. 155-157.

Araucaria. Revista Iberoamericana de Filosofia, Política, Humanidades y Relaciones Internacionales, año 20, ${ }^{\circ} 40$. Segundo semestre de 2018. Pp. 159-184. ISSN 1575-6823 e-ISSN 2340-2199 doi: 10.12795/araucaria.2018.i40.07 
Zhongxian?" ${ }^{10}$. Kang Youwei se inscribía explícitamente en una tradición bien establecida del dang "justo" o "correcto" (zheng), típico de la tradición historiográfica, y se oponía de este modo al dang "injusto" o "desviado" (xie) de la literatura jurídica de la dinastía.

Sin embargo, los reformadores no se limitaron a movilizar las connotaciones tradicionales del dang. También se encargaron de orientarlo en una nueva dirección. Dos elementos resultaron clave en esta reconceptualización. El primero fue la búsqueda de una "coexistencia" con otros partidos. En 1898, durante el período de reformas, este ideal de coexistencia respondía a un esquema ante todo dual, del estilo "Whigs y Tories"; por el momento, el grupo de revolucionarios dirigido por Sun Yatsen no se demarcaba claramente ni del discurso reformador ni de la etiqueta de "bandidos" que, con el vocabulario político tradicional, le solía imponer el discurso mandarinal ${ }^{11}$. En 1898, durante su única audiencia con el emperador Guangxu (1871-1908; r. 18751908), Kang Youwei sugirió que su grupo de reformadores, "nuestro dang", resultaba de una reacción a los "conservadores" (shoujiu zhe). Esta descripción se inscribía en apariencia en la división tradicional entre el dang "correcto" y el dang "desviado": el "desviado" reposaba sobre objetivos ilegítimos, el "correcto" resultaba de la aspiración a una regeneración de la monarquía. Nada sugería en esa entrevista una naturalización de la división entre dang. Pero cuando se la confronta a su discurso sobre los dang, esta descripción parecía inaugurar en China la distinción, entonces familiar en Europa, entre los partidos "progresista" y "conservador". Es lo que Kang Youwei sugería en sus Reflexiones sobre la reforma en Japón, un texto que le presentó al emperador Guangxu durante los "cien días" de reforma. En una nota sobre los partidos políticos, Kang explicaba con claridad cómo los dang podían convertirse en un elemento necesario de la política imperial y cómo lo nuevo y lo antiguo podrían un día coexistir en el paisaje político de una monarquía reformada:

\begin{abstract}
$\mathrm{Al}$ principio de la reforma, existe necesariamente el mal de que lo antiguo y lo nuevo chocan y los dang divididos se atacan mutuamente. A medida que pasa el tiempo desde que la nueva política aplica la reforma, el dang antiguo se transforma y forma parte de lo nuevo. Después de haber cambiado la política, en medio de su renovación, hay necesariamente opiniones divergentes (...). Al buscar durante un largo tiempo, se eliminan las pequeñas diferencias, se toman las grandes coincidencias y se llega a una política de conciliación. Su principio se encuentra por completo en la vigilancia y el cuidado que le proporciona el monarca ${ }^{12}$.
\end{abstract}

${ }^{10}$ Xiansheng nianpu, p. 135.

11 Sobre el concepto de revolución en China, ver Leese, Daniel, "Revolution": Conceptualizing Political and Social Change in Late Qing China," Oriens Extremus 51 (2012), 25-61. Sobre esta división entre "tradicionalistas" y "reformistas" desde 1895, ver Yves Chevrier, "Antitradition et démocratie dans la Chine du premier XX ${ }^{\mathrm{e}}$ siècle. La culture moderne et la crise de 1'État-Nation", in Mireille DelmasMarty y Pierre-Étienne Will (ed.), La Chine et la démocratie, Paris: Fayard, 2007, p. 376.

12 "Riben bianzheng kao", Quanji 4, 242-243. 
Un artículo de Yan Fu en su diario Noticias nacionales (Guowen bao), "Sobre los dang en China", muestra con claridad el trasfondo de la nota de Kang Youwei. Después de enumerar los tipos de dang que existen en Inglaterra, Francia y Japón, este artículo de 1898 explica de la siguiente manera la diferencia entre los dang "chinos" y "occidentales".

Estos tipos de dang [en Europa y Japón] son diferentes de lo que en otros tiempos se llamaba dang en China, y sin embargo los traductores a veces los traducen [con la palabra china] dang. Los denominados dang en China (...) se forman todos a partir de la fuerza de la situación, y no necesariamente a partir del saber de cada uno de ellos; por eso finalmente hay uno que fracasa y no pueden coexistir. Los dang de los occidentales tienen cada uno su saber y su punto de vista (...). Lo que sostiene cada uno de ellos es correcto, y por eso pueden coexistir y no pueden suprimirse mutuamente. Esta es la diferencia entre los dang de China y los de Occidente ${ }^{13}$.

Así el mundo reformador en general, y Kang Youwei en particular, introducía una nueva forma de comprender los clivajes políticos. El objetivo no era deshacerse por completo del partido conservador o, como había sido históricamente el caso, de suprimir las raíces de la división en partidos. El objetivo era, por el contrario, reconfigurar el paisaje político de modo tal que la división de opiniones no representara un riesgo para la existencia de las instituciones monárquicas.

Una segunda novedad del dang reformador tenía que ver con el modo en que echó raíces en la idea moderna de "nación". Los dang anteriores, incluidos los que Kang llamaba "conservadores", se concebían en general como movimientos internos en la corte, y la legitimidad que reivindicaban derivaba de un deber que se imponían ya fuera frente al emperador, ya frente al Cielo. En este discurso, el "pueblo", min, no formaba parte del dang, sino que se reducía a su doble rol tradicional: era tanto testigo como objeto de las acciones del monarca. El pueblo aparecía en el discurso dinástico como "los ojos y oídos del Cielo", metáfora recurrente cuyo origen se encontraba en el libro de los Documentos, una de las escrituras "confucianas" más antiguas. El pueblo aparecía también, en las Analectas, como la hierba que cambia de posición según la dirección viento -una imagen en la que el "viento" simbolizaba las buenas costumbres que el monarca debía cultivar entre sus súbditos. En otras palabras, el pueblo no tenía una existencia política independiente, sino que en teoría debía su bienestar y su moralidad a la acción del monarca y de sus ministros. El dang reformador

13 Yan Fu ji, Beijing: Zhonghua shuju, 1986, 487. La idea del dang reformador como vía media está presente en este artículo, pero sólo como objeto de refutación. Yan Fu sostiene que el partido de Sun Yatsen, relativamente visible y defendido por los "occidentales", no es un verdadero dang. Sólo cuentan según él partido conservador y el partido reformador. La tripartición es en cambio una refracción inducida por una proyección errónea de los observadores externos. 
impuso un discurso diferente. En la inauguración de la Sociedad para Salvar a la Nación (Baoguo hui), que los reformadores fundaron en Pekín en 1898, Kang Youwei no se dirigió a sus pares, a la corte o a algún otro cuerpo del imperio. Se dirigió a los "400 millones de chinos". Los principios de esta Sociedad, inscriptos en su reglamento, explicitaban con claridad la naturaleza de estos "400 millones": eran una "nación" (guo), una "raza" (zhong) ${ }^{14}$ y una "doctrina" (jiao) ${ }^{15}$. La palabra guo, que en su uso tradicional significaba el territorio monárquico, designaba aquí el territorio de un pueblo-raza con su propia "doctrina" y su propia “civilización". Se trataba de un elemento profundamente novedoso en el discurso mandarinal. Apenas treinta años antes, el concepto de nación podía parecer a tal punto extraño que Guo Songtao, embajador de la dinastía Qing con ideas reformadoras, lo tradujo fonéticamente como nashen. En los años 1890, en cambio, la nación correspondía en boca de los mandarines reformadores a una constelación de palabras antiguas que les eran familiares: según el caso, guo o "país" y min o "pueblo" eran ahora sinónimos de los "400 millones de chinos" que habitaban el territorio Qing. Frente a la idea tradicional de que la unidad política de la población tenía su principio en la existencia de un monarca tutelar, estos mandarines reformadores, inspirados del concepto euro-americano de "nación", consideraban los 400 millones no sólo como una suerte de individuo en una sociedad mundial de naciones, sino también, sobre todo, como un sujeto autónomo de las instituciones políticas que lo gobernaban ${ }^{16}$. Esta idea, novedosa en la historia mandarinal del discurso político, les permitió a los reformadores disociar su dang del espacio reducido de la corte y de asociarlo a este nuevo sujeto político.

Esta idea era el principal sostén del dang reformador. El pueblo aparecía como un sujeto autónomo, relativamente independiente de las instituciones

${ }^{14}$ Sobre la idea de "raza" como "nación", ver Dikotter, The Discourse of Race in modern China, Hong Kong : Hong Kong University Press, 1992, p. 97-125. Para Dikotter, la idea de nación estaba vinculada tanto a una la raza como al territorio. Pero la idea de "raza", como muestro en este ensayo, también ha permitido a los reformadores pensar la "nación" fuera de un territorio particular.

15 Chen Baoliang, Zhongguo de hui yu she, Hangzhou: Zhejiang renmin chubanshe, 1996, pp. 67-68. Xiansheng nianpu, 157. La noción de "raza" (zhong) era, en China y en otros lugares, fundamentalmente ambigua y polisémica. Para Kang Youwei, la raza abarca al conjunto de un pueblo unido por lazos de sangre que, no obstante, es más amplio -y por lo tanto incluyente- que los diferentes "grupos étnicos" que lo componen -como los Han y los Manchus. En otras versiones de zhong (por ejemplo, la que se encuentra entre los nacionalistas) la "raza" se reduce al grupo étnico: la "raza china" sería la "raza Han". Sin embargo, en ambos casos, el vocabulario de la "raza" para hablar de las personas y de la nación creó una noción que iba más allá de la separación entre el emperador y sus funcionarios por un lado y el "pueblo" por el otro. En este sentido, la raza contribuyó a abrir el camino a la idea de una "nación soberana" opuesta a la idea de que la soberanía estaba en la única figura del emperador.

${ }_{16}$ Sobre la historia del concepto de nación en el discurso chino, ver Matten, Marc, "China is the China of the Chinese: The Concept of Nation and its Impact on Political Thinking in modern China," Oriens Extremus 51 (2012), 63-106; y Blitstein, Pablo (2016): "A New China in Mexico. Kang Youwei and his Languages of Cohesion-Making on the Two Sides of the Pacific (1895-1911)", Oriens Extremus, 55 (2016), p. 209-260.

Araucaria. Revista Iberoamericana de Filosofía, Política, Humanidades y Relaciones Internacionales, año 20, $\mathrm{n}^{\circ} 40$. Segundo semestre de 2018. Pp. 159-184. ISSN 1575-6823 e-ISSN 2340-2199 doi: 10.12795/araucaria.2018.i40.07 
políticas, y en ese sentido la Sociedad para Salvar a la Nación se presentaba no tanto como figura protectora, sino como intérprete del pueblo-nación. Algunos miembros de la facción conservadora vieron elementos subversivos en esta idea. Según cuenta Liang Qichao, discípulo de Kang Youwei y figura central de la reforma, un censor manchú de la bandera amarilla, Wen Ti, acusó a Kang Youwei de "defender la nación y de no defender a los Gran Qing”. Wen Ti entendía claramente el peligro que el discurso nacionalista representaba para la dinastía reinante: si la nación es algo diferente de la dinastía, o incluso si la dinastía es sólo una parte de esa nación, eso quería decir que la nación podía eventualmente existir sin una dinastía. En ese sentido, proteger la nación, como decía en su acusación, no equivalía a proteger los Gran Qing ${ }^{17}$. Liang Qichao describe esta acusación como una difamación; según explica, ninguno de los que escuchó o leyó en la prensa el discurso inaugural de Kang Youwei tenía duda alguna de su lealtad a la dinastía. Pero aunque es verdad que Kang Youwei siempre defendió la monarquía Qing, incluso después de la fundación de la República, la intuición de Wen Ti no era errada: el dang reformador identificaba un nuevo sujeto político que, tarde o temprano, terminaría por oponerse a la dinastía Qing.

\section{El dang sale de la corte y entra en la haute finance}

Ya a fines del imperio, como hemos visto, dang se impuso a otras palabras para nombrar el concepto de partido político. Las dimensiones ministeriales del dang, su carácter "estatal", sin duda contribuyeron a sincronizar esta palabra con la evolución semántica del partido político en el mundo euro-americano, y el Japón de Meiji, inspiración política e intelectual de reformadores y constitucionalistas chinos, utilizaba también esta misma palabra (pronunciada tô en japonés) para designar sus partidos políticos parlamentarios. Pero dang no tenía un significado puramente textual, de "diccionario". Como otras palabras de la misma constelación semántica -es el caso de hui o "asociación"-, su significado dependía de cada experiencia concreta del dang, textual o no textual; por decirlo de modo más preciso: las representaciones, las imágenes, las proyecciones tenían su fuente en el modo en que los reformadores organizaban, usaban, sentían su dang y el dang de otros. En el caso de muchos reformadores y constitucionalistas que se quedaron en territorio chino después de la represión de 1898, la experiencia del "partido" estaba arraigada en asociaciones (hui y she) de todo tipo, de sociedades nacionalistas a sociedades de poetas, en algunos casos siguiendo patrones nuevos de organización, en otros adoptando formas

\footnotetext{
${ }^{17}$ Liang Qichao, Wuxu zhengbian ji, 76.
} 
más tradicionales ${ }^{18}$. En el caso los reformadores en exilio, como Kang Youwei y su discípulo Liang Qichao, la experiencia del partido tomaba forma en un contexto muy diferente: era la organización de una asociación transcontinental entre el Japón, el Sudeste asiático y las Américas. Después de 1898, el dang fue para ellos la red que lograron constituir alrededor de esta asociación.

La experiencia de la Sociedad para la Salvar a la Nación, novedosa por sus ideas, no lo había sido tanto por su organización interna. En tanto "asociación" (hui), se inscribía en una larga tradición de la China imperial. Con frecuencia fundadas en la iniciativa de una persona prominente, las asociaciones eran en cierto modo instituciones creadas desde "afuera" de la administración, y en ese sentido ofrecían el modelo de un espacio "autónomo" de organización nacional. Es verdad que una buena parte de estas asociaciones $-\mathrm{y}$ la Sociedad no había sido una excepción en este respecto- reposaban sobre mandarines, funcionarios o clanes poderosos de una localidad. Pero en la gran mayoría de los casos, las asociaciones se presentaban como iniciativas independientes. Entre 1895 y 1898, Kang Youwei fundó varias de estas asociaciones. Las dos más importantes, la Sociedad para el Estudio del Autofortalecimiento y la Sociedad para Salvar a la Nación, eran ante todo asociaciones de pares, tanto de gobernadores y ministros como de mandarines sin puesto con diplomas más o menos elevados. En ambos casos, su rol era reclamar reformas desde el llano o sostener el dang reformador que se había echado raíces en la capital y en la corte. La existencia de estas organizaciones dependía de los conflictos políticos internos de la administración imperial. Eran, en cierto modo, un apéndice del dang de la corte.

Esta experiencia asociativa se desarrolló nuevamente en el exilio. Sólo que, alejados de las instituciones imperiales, los reformadores no encontraban, por el momento, ningún dang que sostuviera sus reivindicaciones en la corte. Los reformadores crearon entonces una asociación que, en lugar de ser un apéndice del dang reformador, pasó a identificarse con el dang mismo: es la Sociedad para salvar al Emperador (Baohuang hui), que los reformadores fundaron en Victoria, Canadá, en 1899. "Nuestro dang" - como se decían los reformadores en su correspondencia- adoptó de este modo el nombre de una asociación, hui, que evocaba en su nombre la persona misma del emperador Guangxu. La naturaleza de esta "asociación" es difícil de definir. Concebida como un medio

18 Sobre la historia de los dang, she y hui en China, ver Chen Baoliang, ibid. Sobre la importancia de las asociaciones en el desarrollo de los partidos políticos en China, ver Liu Zehua y Liu Jianqing, "Civic Associations, Political Parties, and the Cultivation of Citizenship Consciousness in Modern China", in Joshua Fogel y Peter Zarrow (ed.), Imagining the People: Chinese Intellectuals and the Concept of Citizenship, 1890-1920, Armonk - London, M. E. Sharpe, 1997, p. 39-60. Esta misma valoración del dang a través del hui es paralela a la valoración de qun, una "sociabilidad" política que, como observa Philip Kuhn, no estaría teñida de faccionalismo. Ver Kuhn, Philip, Les Origines de l'État chinois moderne, Paris, Éditions de l'EHESS, 1999, p. 87 (para el uso de qun en Wei Yuan) y 179 (para qun en Liang Qichao).

Araucaria. Revista Iberoamericana de Filosofia, Política, Humanidades y Relaciones Internacionales, año $20, \mathrm{n}^{\circ} 40$ Segundo semestre de 2018. Pp. 159-184. ISSN 1575-6823 e-ISSN 2340-2199 doi: 10.12795/araucaria.2018.i40.07 
para apoyar a un monarca reformador, sus objetivos parecían mucho más limitados que las ambiciones nacionales de las asociaciones previas; lejos de parecerse al modelo de partido que Kang Youwei había descripto en su historia de Meiji, el dang no sólo se identificaba únicamente a una simple asociación sin vínculo directo con la administración y la corte, sino que además parecía reducirse a un grupo con objetivos de corto plazo. Sin embargo, esta unidad forzada entre dang y hui, creada por las circunstancias del exilio, y sobre todo el hecho de encontrarse fuera de toda institución política, le dieron a la Sociedad para Salvar al Emperador un carácter más amplio y más perenne que el de las frágiles asociaciones de la sociedad imperial. A miles de kilómetros de la corte imperial, lejos de mandarines y de comerciantes bien posicionados que le dieran su apoyo político y financiero, lejos también de los dispositivos de represión del imperio, la Sociedad se procuró medios de acción hasta entonces relativamente desconocidos para muchos reformadores. Entre ellos, uno cobró una relevancia particular: el dinero.

La recaudación de fondos fue en efecto la principal preocupación de Kang Youwei y sus seguidores. Al haber dejado atrás su asidero en las instituciones imperiales, en las redes de mandarines o en los comerciantes o notables que los sostenían, los reformadores en el exilio no tenían otro remedio que buscar los fondos necesarios para sostenerse como organización. De otro modo, ¿cómo financiar los periódicos, los eventos, las reuniones, las celebraciones y las actividades de proselitismo? La colecta de fondos probó ser vital. La Sociedad para Salvar al Emperador tomó entonces la forma de una "empresa" (gongsi) y estableció un reglamento que proponía, además de "proteger al emperador" y de "proteger la nación", proteger también "la industria y el comercio" tanto dentro como fuera de China. La Sociedad consistiría en una sociedad de acciones y obtendría sus recursos de donaciones y de las cuotas que pagaran sus miembros (una pieza de plata americana); esos fondos servirían para constituir un banco y los intereses serían distribuidos entre los accionistas ${ }^{19}$. Esta forma capitalismo financiero fue sin duda un incentivo mayor para los donantes. Pero la Sociedad ofrecía también otro tipo de incentivos: según el monto donado, los dirigentes de la Sociedad elevarían memorias al emperador para que se les otorgara a los donantes títulos y cargos de distinto rango ${ }^{20}$. Este incentivo claramente simbólico -el emperador seguía a fin de cuentas preso- era también un signo de que la Sociedad se consideraba, en cierto modo, como un "estado paralelo" en el exilio que aspiraba a constituirse en el estado legítimo. Los donantes se procuraban, a través de sus inversiones, un lugar en este "estado paralelo" que algún día quizá encontrara su propio territorio.

19 "Baojiu Da Qing huangdi gongsi li", Kang Youwei yu Baohuang hui, 258-260.

20 "Baojiu Da Qing huangdi gongsi li”, Kang Youwei yu Baohuang hui, 260-262. 
En este contexto, los residentes de los Chinatowns del sudeste asiático, Japón y las Américas se convirtieron en el apoyo principal de la Sociedad, y eso obligó a los reformadores a adaptar sus actividades a las expectativas de los grupos y organizaciones de la diáspora. Por un lado, una buena parte de los interlocutores de la diáspora -muchos de ellos cantoneses como Kang Youwei- simpatizó con el objetivo político de la Sociedad: empujar la corte de Qing hacia la reforma. Este interés en los asuntos internos del imperio, muchas veces obtenido con el esfuerzo militante de los reformadores, se inscribía en un proceso de "nacionalización" de la diáspora china ${ }^{21}$. Mientras que durante el siglo diecinueve la diáspora había reproducido en los países anfitriones las divisiones segmentarias entre familias y localidades, y había orientado una buena parte de sus acciones según lealtades familiares y regionales, los reformadores contribuyeron a transformar los lazos de los chinos de ultramar en lazos "nacionales", ligados no ya a localidades, sino a la "China" como totalidad. Por otro lado, la diáspora también confirió a la Sociedad, en continuidad con organizaciones preexistentes, un rol que en cierto modo la alejaba de la política "nacional" china: el papel de mediador frente a las autoridades y los agentes económicos de los países anfitriones. En 1905, por ejemplo, en protesta contra las leyes de exclusión y otras medidas anti-chinas en los Estados Unidos, la Sociedad participó activamente en la organización del boycott de bienes americanos exportados a China. La Sociedad asumió con fuerza el doble rol que la asignaba la diáspora: la de operar tanto en China como en los territorios anfitriones.

La diáspora no fue el único factor en la transformación de las prácticas políticas de los reformadores. Integrados al juego financiero, Kang Youwei y la mayoría de los líderes de la Sociedad adoptaron prácticas que eran necesariamente diferentes de la agitación militante y de la intriga de corte. Varios de ellos tuvieron que aprender los códigos de lo que Karl Polanyi llamó haute finance, una expresión francesa que en la Belle Epoque designaba un área de la sociabilidad de élite creada en torno a los movimientos financieros ${ }^{22}$. Kang Youwei fue en este sentido paradigmático. Reemplazó su mundo de funcionarios y mandarines por hoteles de lujo, residencias de notables, mansiones de empresarios y oficinas de ministros y de presidentes. Por ejemplo,

${ }^{21}$ Sobre la nacionalización de la diáspora, sobre todo en el Nuevo Mundo, y sobre el rol de las organizaciones reformadora y revolucionaria, ver McKeown, Adam, Chinese Migrant Networks and Cultural Change: Peru, Chicago, Hawaii, 1900-1936. Chicago y Londres: The University of Chicago Press, 2001, 86-97; y de modo más general. Armentrout Ma, L. Eve. Revolutionaries, Monarchists and Chinatowns. Honolulu: University of Hawaii Press, 1990. Para una historia de la migración china en México y otras partes de Latinoamérica en general, ver Hu-Dehart, Evelyn, "Coolies and Shopkeepers: The Chinese as huagong (laborers) and huashang (merchants) in Latin-America/ Caribbean," in Anderson, Wanni, and Lee, Robert, Displacements and Replacements. Asians in the Americas, New Brunswick: Rutgers University Press, 2005, 78-111.

${ }^{22}$ Sobre el rol de la haute finance en la época, ver Karl Polanyi, The Great Transformation, Boston, Beacon Press, 2001, 9-20.

Araucaria. Revista Iberoamericana de Filosofía, Política, Humanidades y Relaciones Internacionales, año 20, $\mathrm{n}^{\circ} 40$ Segundo semestre de 2018. Pp. 159-184. ISSN 1575-6823 e-ISSN 2340-2199 doi: 10.12795/araucaria.2018.i40.07 
en ocasión de la visita de Kang Youwei a México DF, el Mexican Herald, un diario mexicano anglófono, presentaba de este modo al visitante:

Su Excelencia Kong Yuwei ha venido a la capital mexicana directamente desde Torreón, donde hay vastos intereses chinos y una no pequeña colonia china. Antes de visitar Torreón, el señor Kong estuvo en muchas de las ciudades más importantes de los Estados Unidos, incluyendo Nueva York, Philadelphia, Washington, St Louis y San Antonio. Ahí habló con mercaderes y financistas representativos sobre el comercio recíproco entre China y los Estados Unidos. Más tarde, en Washington, tuvo una interesante entrevista con el Presidente Roosevelt. El señor Kong se aloja con su Secretario en el Hotel Palacio, donde ocupan la "suite d'honneur" 23 .

En esa "suite d'honneur", Kang Youwei recibió durante unos días a varios notables de la comunidad china local, y después consiguió entrevistarse con el Presidente Profirio Díaz. El objeto central de estas entrevistas era la búsqueda de oportunidades financieras ${ }^{24}$.

La experiencia de la finanza fue fundamental para la evolución de la organización reformadora. Es cierto que el dinero había sido, y todavía era, un tema central en los asuntos de la corte imperial: ya fuera la política impositiva, un tema antiguo en las finanzas dinásticas, ya fuera el crecimiento de la deuda pública, un problema que se agudizaba con el pago de las reparaciones de guerra, ya fueran finalmente las diferentes empresas financiadas desde los años 1860 con fondos imperiales, el dinero ocupaba un lugar central en las discusiones de los reformadores más viejos y más jóvenes. Además, muchos de ellos habían pasado por diferentes actividades lucrativas, en sus localidades o en las ciudades. Sin embargo, había una diferencia entre la experiencia financiera en el exilio y la experiencia que podía tener la corte Qing. En la práctica de los ministros de la corte, el dinero se limitaba a un medio subordinado a las necesidades del imperio: como las deudas corrían el riesgo de reducir la autonomía de la política imperial (como era de hecho el caso), el objetivo era precisamente organizar la circulación de dinero según objetivos políticos; de modo más general, al menos en los medios mandarinales, la práctica del comercio, sin duda fundamental, retenía una cierta dependencia simbólica de la carrera de funcionario imperial: ganar dinero, con todo el poder que eso podía conferirle a una familia, tenía menos valor que embarcarse en la carrera de funcionario. La experiencia de los reformadores en el exilio era diferente. A diferencia de los funcionarios imperiales, que trataban en cierto modo de

\footnotetext{
23 "China is Mexico's Future Customer", Mexican Herald, 28 de junio 1907.

${ }^{24}$ Para más detales sobre la experiencia mexicana de Kang Youwei, y sobre todo su relevancia para los modos en que formulaba su concepto de nación y de raza, ver mi artículo en Oriens Extremus en 2016. En ese momento, aún no conocía el trabajo de Juan Puig ni el ensayo-ficción de Julián Herbert (con elementos tanto ficcionales como históricos) sobre la masacre de chinos en La Laguna.
} 
domesticar el dinero, los reformadores en el exilio identificaron su fuerza política a su fuerza financiera, y en ese sentido orientaron sus recursos a la circulación autónoma y relativamente impredecible del capital financiero. Las finanzas eran repentinamente un espacio político en sí mismo. Y en ausencia de perspectivas de una carrera en el imperio, estos mandarines reformadores abrazaron con entusiasmo el mundo de los negocios y de la especulación.

Kang Youwei pareciera haber traducido esta experiencia en uno de los textos más importantes que escribió en la primera década de 1900: La materia puede salvar a la nación. La palabra "materia" (wuzhi) del título se refería a la industria, la tecnología y las finanzas; era un concepto nuevo que respondía a una nueva experiencia de lo que hoy llamamos "economía" - un concepto en ese entonces hesitante en el mundo sinoparlante. Según entendía, la "materia" era el fundamento de toda nación, de todo estado, de todo ejército; ya no era un producto e instrumento de la estabilidad política (tal como percibía la dinastía las empresas desarrolladas desde los años 1860), sino el fundamento mismo de toda forma de tejido social: "todos los asuntos tienen su fundamento en la materia" ${ }^{25}$. De ahí que en las reglas de la Compañía comercial que Kang Youwei fundó en 1903, y que dependía de la Sociedad para Salvar al Emperador, los bancos aparecen como estructuras autónomas que hacen perdurar las naciones más allá de los estados que las acogen:

Si los Estados [guojia] un día desaparecen, no tendremos motivo para no exigir la subsistencia de los bancos de nuestra Sociedad. Su solidez y dimensiones serán verdaderamente inconcebibles ${ }^{26}$.

La relación entre el dang reformador y la "nación" ya no era la misma que en China. En lugar de la "nación" territorial en la que Kang Youwei había organizado sus asociaciones de reformadores y su facción de corte, su dang reposaba ahora sobre una nación sin territorio "propio" en un sentido político. El espacio de vida de esta nación se encontraba en diferentes partes del mundo; se extendía desde Beijing hasta los barrios chinos de Tokio y Lima. La "nación” se sostenía gracias a logros "materiales" que, según parecía creer, eran asumidos por su Sociedad. La Sociedad tenía entonces un doble papel. Si la China territorial desapareciera de la faz de la Tierra, la Sociedad sería lo que era hasta entonces: una organización de la haute finance que buscaría beneficios para la población china en el extranjero; y si la China territorial sobreviviera, la Sociedad podría volver a ser el dang que, con el emperador Guangxu a la cabeza, lideraría las reformas necesarias para mejorar las condiciones de vida de este "pueblo-raza" en el territorio imperial. El vínculo directo entre dang y "nación" se radicalizó: como el emperador era solo un referente virtual, fueron

25 "Wuzhi jiuguo lun," Quanji, 8: 79.

26 "Zhongguo shangwu gongsi yuanqi fu zhang cheng," in Kang Youwei yu Baohuang hui, 278. 
sobre todo los esfuerzos de la "nación" los que sostuvieron el dang reformista. El dang se presentaba como el representante directo de la "nación".

La experiencia de estos reformadores parecía así seguir -de modo involuntario- un consejo de Montesquieu: "Sería algo muy bueno en la aristocracia si, por alguna vía indirecta, se hace salir al pueblo de su estado de debilidad extrema; así en Génova el banco de San Jorge, administrado en gran parte por los principales del pueblo, le da a este último una cierta influencia en el gobierno"27. Esta relación entre el banco y el gobierno aristocrático es en cierto modo análoga a la relación que la Sociedad para Salvar el Emperador pretendía crear entre ella, la diáspora-nación y las autoridades políticas en China o en América: la participación de la nación tendría lugar sólo de forma indirecta y a través de sus "principales", los dirigentes de esta Sociedadbanco. Kang Youwei se oponía a implementación inmediata de la "política [guiada por] el pueblo (minzheng)", y rechazaba categóricamente la irrupción revolucionaria ${ }^{28}$. En su opinión, el pueblo necesitaba aún figuras tutelares -un grupo de reformadores y un monarca ilustrado- para alcanzar un día el ideal del autogobierno. Los bancos de su dang representaban de este modo un medio "indirecto", y ante todo controlado por los líderes de la Sociedad, de hacer participar la "nación" en el ejercicio del poder.

\section{La consolidación de la "vía media" y la despersonalización de las relaciones políticas}

A diferencia de lo que ocurría en los años 1890, cuando el principal rival era el partido "conservador", la cuestión del "poder popular" se transformó en los 1900 en el problema central de la red reformadora. La razón era el peso creciente de los republicanos y revolucionarios dentro y fuera de esta red, tanto en China como en los distintos lugares donde operaba la Sociedad para Salvar al Emperador. Menos presentes durante la reforma de 1898 y durante los primeros años de vida de la Sociedad, la fundación de la Alianza revolucionaria de Sun Yatsen significó la emergencia de una organización rival en el mismo terreno político de los reformadores: la diáspora y la haute finance. La idea de que el dang reformador representaba la "vía media" en política maduró sobre todo en este período. Opuestos desde un principio a la política "conservadora", los reformadores se vieron obligados a justificarse frente a la emergencia de soluciones más radicales a los problemas que ellos mismos señalaban ${ }^{29}$. En

\footnotetext{
${ }^{27}$ Ver Montesquieu, De l'Esprit des lois, Paris, Flammarion, 1979, vol. 1, p. 136-137.

28 Xiansheng nianpu, p. 408.

${ }^{29}$ Sobre la "vía media", que inscribe a este dang reformador en la "nebulosa" mundial de la reforma de fines del XIX y principios del XX, ver Topalov, Christian, Laboratoires du nouveau siècle. La nébuleuse réformatrice et ses réseaux en France, 1880-1914. Paris, Éditions de l'EHESS, 1999, p. 11-13.
} 
oposición a un miembro de la red reformadora que había adherido públicamente a los métodos revolucionarios, y consciente del peso de esos métodos entre sus propios discípulos -Liang Qichao incluido-, Kang Youwei se vio obligado a escribir en 1902 una carta abierta contra la revolución. La carta es una respuesta dirigida "a los comerciantes chinos de América del Norte y del Sur":

\begin{abstract}
Nuestra Sociedad reposa sobre su extrema lealtad para proteger al emperador, pero el gobierno por el contrario nos considera un partido sedicioso (ni dang), una sociedad de bandidos (fei hui) (...) Después de la furia [que el gobierno Qing provoca con sus políticas], ustedes desean precipitadamente hacer una revolución e independizarse. ¿Acaso no piensan en nuestro sabio soberano, que sacrificó su persona para salvar a nuestro pueblo? Esto no sólo es contrario a las ideas de nuestra Sociedad para Salvar al Emperador, sino que, después de haber reflexionado sobre nuestro tiempo y haber medido las fuerzas, pienso que esto no es posible.... ${ }^{30}$.
\end{abstract}

En su oposición tanto a los conservadores como a los revolucionarios, la Sociedad para Salvar al Emperador se alejaba de la estructura dual entre un dang "justo" y un dang "injusto" y se descubría, sin proponérselo, como una "vía media" entre los dos extremos que dominaban la política "nacional" china entre Asia y América. En esta coyuntura en que los "conservadores" dominaban la corte y las instituciones del imperio, la idea de una vía media estaba ligada a una doble experiencia entre los barrios chinos y la alta finanza, es decir, a una experiencia fuera de toda institución estatal.

Una buena parte de los reformadores insistían en que la nación china no estaba lista para gobernarse a sí misma. En esto coincidían con muchos argumentos políticos en Europa, Asia y las Américas, ya fuera en el campo del sufragio (censitario) o, de manera más profunda, en las discusiones sobre el grado de participación de las poblaciones en las decisiones políticas: el carácter gradual de las reformas debía corresponderse con el proceso de maduración que la nación necesitaba antes de alcanzar el ideal del autogobierno. En principio, esta concepción tutelar de su propio dang se oponía al tipo de construcción de política sobre el que la Sociedad para Salvar al Emperador había reposado hasta el momento. Lejos de ser una organización de mandarines mejor o peor posicionados en las instituciones imperiales, la Sociedad recibía todo tipo de categorías sociales, desde trabajadores a empresarios, y contaba con la participación activa de esta "nación" que ya nada tenía que ver con la corte, el imperio, el mandarinato o algún otro de los cuerpos que dirigían la política de los Qing. Pero los reformadores insistían en el papel tutelar que debían cumplir. Esto se debía quizá a dos razones. La primera era que sus dirigentes más influyentes, como Kang Youwei y Liang Qichao, eran o habían sido formados

\footnotetext{
30 "Da Nan Bei Meizhou zhu huashang lun Zhongguo ke xing lixian, bu ke xing geming shu", Kang youwei zhenglun ji, Beijing, Zhonghua shuju, 1981, p. 473-4.
} Segundo semestre de 2018. Pp. 159-184. ISSN 1575-6823 e-ISSN 2340-2199 doi: 10.12795/araucaria.2018.i40.07 
como mandarines, es decir, como custodios del saber heredado de los Sabios antiguos y como maestros que debían educar a la población para preservar el orden imperial. La segunda razón estaba ligada a los vínculos de los dirigentes reformadores con la haute finance. El mundo de las finanzas, que en cierto sentido se encontraba por encima de la "política" (aunque la finanza fuera en el fondo uno de los medios privilegiados de hacer política dentro o fuera de un estado), no era un mundo "democrático". Si bien en China el mundo del comercio podía usar sus propios medios de presión para exigirle a la dinastía una extensión del "poder popular", en el Sudeste asiático o en los Estados Unidos la Sociedad funcionaba como una compañía comercial en la que sus dirigentes decidían la dirección de las inversiones y en que los trabajadores chinos de las distintas empresas en que se embarcaba no tenían decisión alguna sobre la política financiera. En otras palabras, mandarines y hombres de negocios coincidían en una posición tutelar no sólo frente a los trabajadores, sino frente a los miembros mismos de la Sociedad. En un pasaje de su Libro de la Gran Unidad que no es posible datar con precisión, pero que remonta sin duda a esta experiencia político-financiera, Kang Youwei deja entrever el rol "mandarinal" que le atribuye a sus experiencias empresariales y las homologías entre los mundos mandarinal y financiero:

En los tiempos de la Gran Unidad, la industria será pública, y su tamaño será algo que nuestra época no podría concebir: su personal será de miles de millones, su territorio de cientos de miles de $l i$; en su interior su territorio será como el de los antiguos estados, sus gestores serán como los monarcas de los antiguos principados;.. los que conservan los registros serán como grandes mandarines (dafu, "doctores"), sus administradores serán como mandarines (shi), sus capataces serán como mandarines de bajo rango (xia shi) y los trabajadores serán como el pueblo. El departamento donde se discutirá sobre el trabajo será como la corte (chaoting $)^{31}$.

La experiencia de la haute finance y de la diáspora produjo dos cambios fundamentales en el dang reformador: por un lado, el abandono definitivo de la corte como espacio político; por otro, la despersonalización de las relaciones políticas. El segundo cambio fue quizás la consecuencia del primero. La lejanía de la corte había forzado al dang reformador a redefinirse a sí mismo en términos "nacionales" y a pasar por el poder del dinero para organizarse; lejos del territorio imperial, la Sociedad para Salvar al Emperador repentinamente se encontró más cerca de una "nación china" transcontinental y de las finanzas de la Belle Epoque por un lado, y lejos del emperador y de su corte por el otro. Paradojalmente, a pesar de que esta experiencia política estaba marcada por la distancia al territorio chino y, sobre todo, a las instituciones del imperio, los

\footnotetext{
${ }^{31}$ Kang Youwei, Quanji, vol. 7, p. 160.
} 
reformadores sintieron que habían creado un partido político análogo a los que existían en Estados Unidos y Europa occidental. En 1906, Kang Youwei afirmó: "Hoy la China no tiene aún un partido político (zhengdang, lit. "dang político"); nuestro partido es el primero" 32 . En esta percepción, la despersonalización de las relaciones políticas, resultado de esta distancia, pareciera haber jugado un rol central.

Los reformadores habían tenido hasta entonces dos percepciones diferentes de su propio dang. Con el emperador, el dang buscaba establecer o reestablecer una relación personal que le permitiera a sus principales figuras actuar como consejeros; con la "nación", en cambio, la relación sólo podría ser impersonal. Es verdad que algunos reformadores, especialmente Kang Youwei, operaban en los "lobbies" de manera no muy diferente a como actuaba en la corte; la obtención de dinero y las posibilidades de negocios se presentaban muchas veces como resultado de lazos de amistad o de redes de conexiones personales. Pero en el discurso político, estas relaciones quedaban en la oscuridad del modus operandi; lo que quedaba en primer plano del discurso, lo que era a la vez el interlocutor figurado y el beneficiario ficticio de la acción política, era la entidad impersonal de la "nación". Esta despersonalización del interlocutor llevó a los dirigentes reformadores a repensar su discurso y el rol de la Sociedad: ¿los reformadores eran el "dang del emperador” o el "dang de la nación"? La respuesta a esta pregunta estuvo estrechamente ligada a las transformaciones políticas en China. En 1901, después los estragos de la Guerra de los Boxer, la dinastía Qing anunció una "Nueva Política" (o "Renovación de la política”, xinzheng) de reformas educativas, administrativas, jurídicas y fiscales; en 1906, como parte de esta reforma, la dinastía Qing prometió una constitución y un parlamento. Estas reformas provocaron una serie de intercambios epistolares entre Kang Youwei y Liang Qichao, los dos dirigentes principales de la Sociedad para Salvar al Emperador. En sus intercambios, los dos estaban de acuerdo en que deberían cambiar el nombre de su dang; les parecía que era ésta una reacción natural y necesaria frente al hecho, positivo, de que los Qing estaban poniendo en marcha muchas de las reformas que los reformadores propusieron en 1898. Kang anunció entonces, de manera unilateral, que la Sociedad se llamaría Sociedad para la Constitución Nacional (Guomin Xianzheng hui); y como el hui y el dang coincidían en esta única organización reformadora, también lo llamó Partido para la Constitución Nacional (Guomin Xianzheng dang). Según él, este nombre tenía en cuenta el papel activo y la "responsabilidad de los ciudadanos" (guomin zhi ze), que era prepararse para la participación en el parlamento y para gobernar "juntos" con el emperador ${ }^{33}$. Pero Liang Qichao lo convenció de reemplazar la palabra "nacional"

\footnotetext{
32 "Xing qing gai hui jianyao zhangcheng", Kang youwei zhenglun ji, Beijing, Zhonghua shuju, 1981, p. 603.

${ }^{33}$ Xiansheng niangpu, p 396. Voir aussi "Bu gao bai qi shi yu bu hui zhong ding wei xin nian yuan dan ju da qingdian - gao cang baohuang hui gai wei guomin xianzheng hui”, in Kang Youwei zhenglun ji, p. 597. La palabra guomin, lit. "pueblo del reino", era en el discurso reformador (como en Segundo semestre de 2018. Pp. 159-184. ISSN 1575-6823 e-ISSN 2340-2199 doi: 10.12795/araucaria.2018.i40.07
} 
por "imperial" (diguo) y de utilizar el nombre que finalmente llevó la asociación de reformadores: Sociedad para la Constitución Imperial (Diguo xianzheng hui). $\mathrm{Su}$ argumento era que un dang, que en su carta identificó con los zhengdang o "partidos políticos", tenía que permanecer fiel a un "principio fundamental" o "-ismo" (zhuyi), que en el caso de ellos había sido el principio monárquico:

En todos los países del Este y del Oeste, los que hablan de los partidos políticos (zhengdang) [se refieren] a los principios: decimos que hacemos un dang con un principio, no decimos que hacemos un dang con una persona ... ${ }^{34}$.

El "principio" al que hacía referencia era la "constitución imperial": la defensa de una monarquía reformada era desde sus orígenes el principio unificador del dang, y la organización debería recordarlo en su nombre mismo. El uso de la palabra zhengdang, que perduró hasta hoy en día como traducción de "partido político", respondía quizá a su voluntad de despegar su organización de una simple asociación o incluso del antiguo discurso imperial sobre el dang; quizá respondía también al hecho de que zhengdang ya era una expresión corriente para conceptualizar este nuevo fenómeno del paisaje político chino. Pero lo más importante no es el uso mismo de esta palabra, sino la comparación explícita que establece con otros "partidos" en el resto del mundo y las reflexiones politológicas que avanza en su carta. Para Liang Qichao, este hui, el dang o el zhengdang (la Sociedad era los tres al mismo tiempo) se basaba en dos principios fundamentales: la despersonalización de las relaciones políticas y el establecimiento de un programa. En su propuesta, la palabra "imperio" - o más precisamente "nación con emperador", diguoindicaba el apoyo a una forma impersonal de organización nacional, y no sólo una defensa del emperador Guangxu, como había sido el caso hasta ese entonces; la nueva Sociedad afirmaba un principio "conservador" en el sentido de que se mostraba como explícitamente monarquista (en oposición abierta a los republicanos), pero al mismo tiempo renovaba su presentación al poner en escena una relación impersonal y programática con la "nación". Y este principio impersonal, según Liang Qichao, debía reproducirse en la estructura interna del dang.

[Es porque] usted, Maestro, promueve estos principios [de reforma] que [las personas] quieren estar en el mismo dang con usted; no es porque estos principios salgan de usted que quieren estar en el dang [para apoyar] estos principios (...). ${ }^{35}$

el vocabulario de Meiji) la traducción de "ciudadanos" o "ciudadanía"

${ }^{34}$ Xiansheng nianpu, p. 403. La palabra dang aparece en este pasaje utilizada como un verbo. Eso muestra que el hecho de asociarse a un dang es percibido como una acción social específica, distinta de otras formas de acción.

${ }^{35}$ Xiansheng nianpu, p. 403.

Araucaria. Revista Iberoamericana de Filosofia, Politica, Humanidades y Relaciones Internacionales, año 20, $\mathrm{n}^{\circ} 40$. Segundo semestre de 2018. Pp. 159-184. ISSN 1575-6823 e-ISSN 2340-2199 doi: 10.12795/araucaria.2018.i40.07 
En otras palabras, el dang debe reposar sobre la impersonalidad del principio que reúne al grupo. Esa eliminación de los vínculos personales en el nombre y en el discurso de la Sociedad no significaba que estos vínculos personales ya no serían necesarios, sino que quedarían necesariamente ocultos. Las viejas dependencias personales, en especial la relación maestro-discípulo que existían entre sus dirigentes, servirían para cimentar las relaciones entre los miembros. Así,

...cuando uno se deja guiar por estos principios, nadie más que usted puede comandar como un líder ${ }^{36}$.

Este juego entre lo oculto y lo visible, entre lo personal en la práctica y lo impersonal en el discurso, era homólogo a la idea misma de la organización del "Estado" monárquico chino. En la China reformada, la constitución monárquica representaba los fundamentos impersonales de la nación, y el monarca el fundamento personal necesario para implementar los principios de la constitución; la constitución representaba el consenso, el monarca la figura tutelar de la nación ${ }^{37}$. En el dang reformador, análogo a un "estado" reformado, el programa sería como la constitución y Kang Youwei el maestro oculto, la figura tutelar, el "monarca". Los "principios" se convertirían en el fundamento "visible" del dang y del estado, la persona física del maestro y del monarca serían las fuerzas "ocultas" que le darían vida a uno y otro. Liang Qichao lo dice explícitamente: las líneas directivas (zhangcheng) de la asociación (hui) son como la constitución (xian) de un país. Las representaciones de la organización interna del dang eran homólogas a las representaciones sobre la organización del estado-nación ${ }^{38}$.

Este principio impersonal de organización, y sobre todo el énfasis en el carácter impersonal de la "constitución", contribuía a transformar el dang en una organización que trascendía la generación de sus fundadores. Aunque Kang seguía siendo el líder supremo del partido y las instituciones imperiales suponían la existencia de la persona física del monarca, los fundamentos impersonales le daban al dang la capacidad de durar más allá de Kang Youwei, más allá de los líderes, más allá la persona del emperador -más allá de los vivos. Esta fue una ruptura discursiva adicional con el dang de corte. Los dang

\footnotetext{
${ }^{36}$ Xiansheng nianpu, p. 403.

${ }^{37}$ La impersonalización de las relaciones políticas era, por el momento, discursiva: se aplicaba al discurso, pero no se aplicaba a la relación entre los miembros del dang. Kang Youwei todavía era el "maestro", Liang Qichao y los otros, los "discípulos". Incluso en este momento de reformulación del nombre del dang y de la voluntad de fundar un "partido de ideas", la asociación entre ellos no siguió el principio de "asociación libre" en función de las propias convicciones (lo que permite derrocar las jerarquías y reducir el maestro a un simple individuo portador de ideas), sino el principio de una jerarquía personal.

38 Xiansheng nianpu, p. 403.
} 
"facciosos" del imperio habían sido concebidos como grupos generacionales cuya existencia debía cesar una vez que un emperador sabio restableciera la armonía. En Kang Youwei y Liang Qichao, en cambio, la experiencia de la desterritorialización produjo otra forma de dang: la de un grupo de presión destinado a permanecer más allá de los gobernantes y las instituciones, y cuya permanencia a través de las generaciones estaba asegurada por la existencia misma de una "nación china" con una historia propia que reúne a los vivos, los muertos y los que están por venir. El anclaje "nacional", ya fuera bajo la forma de un "imperio territorial" o de un "pueblo sin Estado", había transformado el dang de un "partido de personas" en un "partido de ideas" destinado a durar tanto como la nación china existiera sobre la tierra.

Con esta distinción entre lo personal y lo impersonal, el partido reformista se estaba preparando para ocupar un lugar permanente, transgeneracional, en las instituciones parlamentarias de una monárquía constitucional. En 1911, en los albores de la revolución en China, Kang Youwei recapitulaba de este modo la evolución de su dang:

China no tuvo partido político (zhengdang) durante miles de años; después de la derrota en la Guerra con el Japón, empezamos a promover uno en la capital, bajo el nombre de Sociedad para el Estudio del Auto

fortalecimiento (...) En 1898, lo abrimos y lo agrandamos bajo el nombre de Sociedad para Salvar a la Nación y lo extendimos a cada provincia (...) En 1899 lo abrimos al extranjero y pasó a llamarse Sociedad para Salvar al Emperador. Cuando en 1906 se promulgó el edicto para establecer una constitución, le cambiamos el nombre y le pusimos Sociedad para la Constitución Imperial (...). La constitución es el padre del partido; el parlamento, su madre (...). Cuando constitución y parlamento convergen, es imposible evitar el nacimiento de los partidos. ${ }^{39}$

Como lo había sugerido unos años antes en su historia de las reformas de Meiji, Kang Youwei aspiraba a colocar los conflictos dentro de una coexistencia pacífica entre partidos. La posibilidad misma de distinguir entre lo transitorio y lo permanente en las instituciones políticas, entre una institución basada en un conflicto político controlado (los partidos) y una institución política que se encontrara por encima del conflicto político (la constitución monárquica), surgió precisamente del doble juego entre lo impersonal y lo personal. Si la oposición impersonal entre los "principios" de los diversos dang debía ser el espacio sublimado del conflicto controlado y de la coexistencia de opiniones (en particular en un parlamento), la persona del monarca y sus herederos debían portarse garantes de una "constitución" que se encontrara, al menos en teoría, por encima de los conflictos cotidianos que dan origen a los partidos ${ }^{40}$.

39 "Minzheng bu zhun Diguo tongyi dang zhuce lun", Kang Youwei yu Baohuang hui, 314.

${ }^{40}$ Es precisamente la idea que opuso a los conceptos de guoti, "cuerpo nacional", a zhengti, "cuerpo 
De la facción de corte a la asociación transcontinental, de la organización financiera al partido proto-parlamentario, el dang cobró formas diversas a medida que los reformadores lo hicieron renacer en contextos diferentes. Este término tuvo distintos significados a lo largo de la historia imperial, y en ese sentido, nada diferenciaba esta evolución conceptual de otras evoluciones previas. Pero la convergencia entre la experiencia mandarinal, la experiencia del activista exiliado y, ante todo, la experiencia de la haute finance le dieron al dang reformador una doble cara. Por un lado, la lejanía de los dispositivos represivos de la dinastía, la relativa libertad de acción y la relación estrecha con una "nación" transcontinental le dieron a los reformadores la oportunidad de presentarse como un verdadero "partido nacional" que representaba, ante todo, las ideas de "los de abajo"; por otro lado, las dependencias personales "ocultas", el modus operandi del mundo financiero y los reflejos mandarinales de los dirigentes sentaron las bases de un partido tutelar que le negaba a la "nación" su capacidad de gobernarse a sí misma. Este dang reformador adoptaba así, paradojalmente, algunas de las características del dang "conservador"; aunque pretendía ser el representante de una "nación" activa, por el momento se negaba a ofrecerle a este nuevo sujeto político una participación directa en las instituciones. De este modo, a través de argumentos que quedarían disponibles en el lenguaje partidario del siglo 20, los principales dirigentes reformadores, miembros tanto del mandarinato como de la "aristocracia financiera" de la Belle Epoque, contribuyeron a consolidar una forma tutelar de partido que marcaría el paisaje político de fines del imperio y de la República.

\footnotetext{
político": la "nación china" parecería tener fundamentos que requieren ciertas formas institucionales específicas. Una vez la monarquía abolida, Liang Qichao tomó distancia del monarquismo y se dedicó a defender la estabilidad institucional, ya fuera republicana o monárquica. Así, por ejemplo, inspirado por Fichte, en 1915 escribió un texto (sin terminar) en el que proponía un guoti donde el egoísmo se traduciría en altruismo, porque la única manera de lograr las aspiraciones individuales sería en cooperación con los demás. Aquí, el papel de los hombres talentosos sería guiar a aquellos con menos talento hacia el objetivo final de lograr el bien común. Las relaciones "nacionales" aparecen en este texto como definitivamente impersonales, y las instituciones políticas deberían reposar sobre ellas. Ver Joachim Kurtz, "Translating the Vocation of Man: Liang Qichao (1873-1929), J.F. Fichte, and the Body Politic in Early Republican China", in Martin Burke y M. Richter (éds), Why Concepts Matter: Translating Social and Political Thought, Leiden-Boston: Brill, 2012, 153-175.
} 


\section{Referencias bibliográficas:}

Armentrout Ma, L. Eve. Revolutionaries, Monarchists and Chinatowns. Honolulu: University of Hawaii Press, 1990.

Blitstein, Pablo, "A New China in Mexico. Kang Youwei and his Languages of Cohesion-Making on the Two Sides of the Pacific (1895-1911)", Oriens Extremus 55 (2016), pp. 209-260.

Brunner, Otto, Werner Conze, y Reinhart Koselleck (eds.), Geschichtliche Grundbegriffe. Historisches Lexikon zur politisch-sozialen Sprache in Deutschland, Stuttgart: Klett-Cotta, 1972-1997.

Chen Baoliang, Zhongguo de hui yu she, Hangzhou: Zhejiang renmin chubanshe, 1996.

Chevrier, Yves, "Antitradition et démocratie dans la Chine du premier $\mathrm{XX}^{\mathrm{e}}$ siècle. La culture moderne et la crise de l'État-Nation", in Mireille Delmas-Marty y Pierre-Étienne Will (eds.), La Chine et la démocratie, Paris: Fayard, 2007.

"China is Mexico's Future Customer", Mexican Herald, 28 de junio 1907.

Dikötter, Frank, The Discourse of Race in Modern China, Hong Kong: Hong Kong University Press, 1992.

Guilhaumou, Jacques, "De l'histoire des concepts à l'histoire linguistique des usages conceptuels", Genèses, n 38, 2000, pp. 105-11.

Hu-Dehart, Evelyn, "Coolies and Shopkeepers: The Chinese as huagong (laborers) and huashang (merchants) in Latin-America/Caribbean," in Anderson, Wanni, and Lee, Robert, Displacements and Replacements. Asians in the Americas, New Brunswick: Rutgers University Press, 2005, pp. 78-111.

Jiang Yihua y Zhang Ronghua, Kang Youwei quanji, Beijing: Zhongguo renmin daxue chuban she, 2006. 12 vols.

Kuhn, Philip, Les Origines de l'État chinois moderne, Paris, Éditions de l'EHESS, 1999.

Kurtz, Joachim, "Translating the Vocation of Man: Liang Qichao (1873-1929), J.F. Fichte, and the Body Politic in Early Republican China", in Martin Burke y M. Richter (eds.), Why Concepts Matter: Translating Social and Political Thought, Leiden-Boston: Brill, 2012, pp. 153-175.

Leese, Daniel, “'Revolution”: Conceptualizing Political and Social Change in Late Qing China," Oriens Extremus 51 (2012), pp. 25-61.

Levine, Ari D., Divided by a Common Language: Factional Conflict in Late

Northern Song China, Honolulu: University of Hawaii Press, 2008.

Liang Qichao, Wuxu zhengbian ji, Shanghai: Zhonghua shuju, 1954. 
Liu Zehua y Liu Jianqing, "Civic Associations, Political Parties, and the Cultivation of Citizenship Consciousness in Modern China", in Joshua Fogel y Peter Zarrow (ed.), Imagining the People : Chinese Intellectuals and the Concept of Citizenship, 1890-1920, Armonk-London: M. E. Sharpe, 1997.

Matonti, Frédérique, "Plaidoyer pour une histoire sociale des idées politiques", Révue d'histoire moderne et contemporaine, vol. V, n 59, 2012, p. 85-104.

Matten, Marc, "China is the China of the Chinese: The Concept of Nation and its Impact on Political Thinking in modern China," Oriens Extremus 51 (2012), pp. 63-106.

McKeown, Adam, Chinese Migrant Networks and Cultural Change: Peru, Chicago, Hawaii, 1900-1936. Chicago y Londres: The University of Chicago Press, 2001.

Montesquieu, De l'Esprit des lois, Paris : Flammarion, 1979.

Murata Yujiro, "Dynasty, State, and Society: The Case of Modern China," in Joshua Fogel y Peter Zarrow, Imagining the People: Chinese Intellectuals and the Concept of Citizenship, 1890-1911, Armonk: M. E. Sharpe, 1997, pp. 113-141.

Polanyi, Karl, The Great Transformation, Boston: Beacon Press, 2001.

Richter, Melvin, The History of Political and Social Concepts: A Critical Introduction, New York- Oxford: Oxford University Press, 1995.

Shanghai shi wenwu bao guan weiyuan hui (ed.), Kang Youwei yu Baohuang hui, Shanghai: Shanghai Renmin chubanshe, 1982.

Tang Zhen, Qianshu jiaozhu, Changsha: Yuelu shushe, 2010.

Tang Zhijun (ed.), Kang youwei zhenglun ji, Beijing: Zhonghua shuju, 1981.

Topalov, Christian, Laboratoires du nouveau siècle. La nébuleuse réformatrice et ses réseaux en France, 1880-1914, Paris: Éditions de l'EHESS, 1999.

Wang Shi (ed.), Yan Fu ji, Beijing: Zhonghua shuju, 1986. 5 vols.

Wu Tianren, Kang Youwei xiansheng nianpu, Taipei: Yiwen yinshu guan, 1994. Zhao Erxun et al. (ed.), Qing shi gao, Beijing: Zhonghua shuju, 1977. 